\title{
Prevalência de alterações visuais entre caminhoneiros no Estado do Pará no ano de 2018
}

\author{
Prevalence of visual disorders among truck drivers in the state of Pará in the year of 2018
}

\author{
Prevalência de alteraciones visuales entre camioneros en el Estado del Pará el año de \\ 2018
}

Rafael Scherer ${ }^{1 *}$, Giovanni Vielmond Borges da Silva², Ana Caroline Coelho Gomes ${ }^{3}$, Marcio Lima da Costa ${ }^{1}$, Vivian Danielle Bastos da Silva Gonçalves ${ }^{3}$, Nathalia Pereira Scherer ${ }^{4}$, Willian Brendon Ferreira Santos ${ }^{2}$, Raissa Tereza Casseb Oliveira ${ }^{1}$, Nicholas Fernandes Mota ${ }^{5}$, Luiz Ricardo Cruz Neves ${ }^{1}$.

\section{RESUMO}

Objetivo: Estudar e quantificar a acuidade visual dos motoristas de veículos pesados atendidos no projeto comando de saúde das rodovias no Pará. Métodos: Estudo epidemiológico retrospectivo, com abordagem quantitativa, descritivo com corte transversal. Constituído através de levantamento em Banco de Dados relativo ao Comando de Saúde em Rodovia Federal do Estado do Pará no ano de 2018, realizado e disponibilizado pela Superintendência de Polícia Rodoviária Federal no Estado do Pará. Foram analisados 185 prontuários. Resultados: Considerando-se a acuidade visual (AV) medida com a melhor correção disponível no momento da direção veicular, para cada olho separadamente, 137 (74,05\%) pacientes tinham AV 20/30 ou melhor no olho direito (OD), e 135 (72,9\%) tinham AV 20/30 ou melhor no olho esquerdo (OE), considerando assim o suficiente para um motorista profissional. Em 18,9\%, a AV foi inferior a 20/40 no olho direito e em $20,54 \%$ no olho esquerdo, incapacitando segundo a legislação para a direção profissional. Somado a isso, 23,8\% demonstraram alteração no teste para estereopsia. Conclusão: A baixa acuidade visual é bastante prevalente entre motoristas profissionais, podendo estar ligadas ao grande número de acidentes no trânsito em rodovias no Estado do Pará.

Palavras chaves: Acuidade Visual, Transtornos da visão, Exame para habilitação de motoristas.

\footnotetext{
ABSTRACT

1 Universidade Federal do Pará - Hospital Bettina Ferro de Souza. Belém - Pará

2 Polícia Rodoviária Federal. Belém - Pará.

${ }^{3}$ Centro Universitário do Estado do Pará (CESUPA). Belém - Pará

${ }^{4}$ Universidade do Estado do Pará (UEPA). Belém - Pará

${ }^{5}$ Associação Brasileira de Medicina do Tráfego (ABRAMET). Belém - Pará.

*E-mail: rafaelscherer.stm@gmail.com
}

Objective: To study and quantify the visual acuity of the heavy vehicle drivers assisted in the highway health command project in Pará. Methods: A retrospective epidemiological study with a descriptive and descriptive cross-sectional approach. Constituted through a survey in a database on the Health Command in the Federal Highway of the State of Pará in the year 2018, made and made available by the Superintendency of Federal Highway Police in the State of Pará. Results: Considering the visual acuity (AV) measured with the best correction available at the moment of the vehicular direction, for each eye separately, $137(74.05 \%)$ patients had $A V 20 / 30$ or better in the right eye (OD), and 135 (72.9\%) had AV 20/30 or better in the left eye (OE), thus considering enough for a professional driver. In $18.9 \%$, AV was less than $20 / 40$ in the right eye and $20.54 \%$ in 
the left eye, disabling according to the legislation for professional direction. In addition, $23.8 \%$ demonstrated a change in the test for stereopsis. Conclusion: Low visual acuity is quite prevalent among professional drivers, and may be related to the large number of traffic accidents on highways in the State of Pará.

Key words: Visual Acuity, Vision Disorders, Automobile Driver Examination.

\section{RESUMEN}

Objetivo: Estudiar y cuantificar la agudeza visual de los conductores de vehículos pesados atendidos en el proyecto comando de salud de las carreteras en Pará. Métodos: Estudio epidemiológico retrospectivo, con abordaje cuantitativo, descriptivo con corte transversal. En el año 2018, realizado por la Superintendencia de Policía Rodoviária Federal en el Estado de Pará, se analizaron 185 fichas de registro de datos sobre el Comando de Salud en Carretera Federal del Estado de Pará. Resultados: Considerando la agudeza visual (AV) medida con la mejor corrección disponible en el momento de la dirección vehicular, para cada ojo por separado, 137 (74,05\%) pacientes tenían AV 20/30 o mejor en el ojo derecho (OD), y 135 (72,9\%) tenían AV 20/30 o mejor en el ojo izquierdo (OE), considerando lo suficiente para un conductor profesional. En el 18,9\%, la AV fue inferior a 20/40 en el ojo derecho y en el 20,54\% en el ojo izquierdo, incapacitando según la legislación para la dirección profesional. Al sumarse a ello, el 23,8\% mostró alteración en la prueba para estereopsia. Conclusión: La baja agudeza visual es bastante prevalente entre conductores profesionales, pudiendo estar ligadas al gran número de accidentes en el tránsito en carreteras en el Estado de Pará.

Palabras clave: Agudeza Visual, Trastornos de la Visión, Examen de Aptitud para la Conducción de Vehículo.

\section{INTRODUÇÃO}

No Brasil, de acordo com os dados do Seguro Obrigatório de automóveis (DPVAT), o número de mortes por acidente de trânsito aumentou de 33.547 no ano de 2016, para 41.151 no ano de 2017, totalizando um adicional de $23 \%$. Estudos apontam que destes acidentes nas rodovias, os veículos com transporte de carga são responsáveis por $25 \%$ dos acidentes, e que $15 \%$ destes causam óbito e $7 \%$ levam a caso de invalidez permanente. (RIBEIRO CLS et al, 2017)

Enxergar bem é uma capacidade essencial para quem quer dirigir, sendo assim, motoristas com boa acuidade visual dirigem com mais segurança e tem menos risco de provocar acidentes. A baixa acuidade visual é o déficit mais comum entre motoristas jovens, sendo importante fator de risco para segurança nas estradas. $O$ estilo de vida pouco saudável e a sobrecarga de trabalho desses profissionais interferem diretamente no cuidado à saúde ocular e a prevenção de novas comorbidades. (BRANDÃO EO et al, 1995)

Segundo a resolução 425/2012 do Conselho Nacional de Trânsito (CONTRAN), para estar apto à conduzir veículos automotores nas categorias C, D e E, é exigido acuidade visual de 20/30 no melhor olho e 20/40 no outro olho, com visão binocular de 20/25, além de que devem ser capazes de reconhecer as luzes semafóricas (verde, vermelho e amarelo) em posições padronizadas, sendo exigido ao candidato possuir visão em baixa luminosidade. Esses valores podem ser obtidos com ou sem correção óptica, devendo, neste último caso, constar na Carteira Nacional de Habilitação $(\mathrm{CNH})$ obrigatório o uso de "lentes corretivas".

É importante atentar-se que nas categorias C, D e E, não é permitido visão inferior a 20/30 em qualquer olho, e os portadores de visão baixa (inferior a 20/40) ou de visão monocular (acuidade 0 - zero) não podem exercer atividade remunerada. (DUARTE WR et al, 2002)

A legislação brasileira de trânsito prevê que a cada 5 anos sejam renovados os exames psicológicos e médicos exigidos aos condutores que exercem atividade remunerada, entre eles, o exame de acuidade visual. Estudos comprovam que esses profissionais devem possuir uma acuidade visual mínima que forneça condições de localizar e reagir a pedestres, veículos e sinalizações. Sendo informado que $44,2 \%$ dos condutores que possuíam comorbidades tiveram complicações em seu campo visual (QUAGLIATO LB et al, 2012). 
Diante deste cenário, os acidentes automobilísticos apresentam importante posição de destaque no aumento da mortalidade por causas externas, e o aumento dos casos de acidentes de tráfego, grande parte provocadas por motoristas profissionais, é um fator de grande relevância para a criação deste trabalho.

O presente estudo tem como objetivo avaliar e descrever a prevalência de alterações visuais entre caminhoneiros no Estado do Pará no ano de 2018, sendo de extrema importância para a criação de novas medidas preventivas e corretivas para evitar tais acontecimentos.

\section{MÉTODOS}

Estudo epidemiológico retrospectivo, com abordagem quantitativa, descritivo com corte transversal. Constituído através de levantamento em Banco de Dados relativo ao Comando de Saúde em Rodovia Federal do Estado do Pará no ano de 2018, realizado e disponibilizado pela Superintendência de Polícia Rodoviária Federal no Estado do Pará.

O Comando de Saúde nas Rodovias é realizado 04 vezes ao ano por cada superintendência estadual da Polícia Rodoviária Federal, em um ponto pré-estabelecido de uma das rodovias que entrecorta o estado. Nestes comandos são atendidos como meta 100 caminhoneiros que trafegam nas rodovias e se dispõem a participar voluntariamente do evento. A partir do aceite do caminhoneiro, o mesmo trafega entre diversas estações de atendimento, onde recebe avaliação multidisciplinar que inclui dados sobre acuidade visual, visão de cores e estereopsia.

Visto que a coleta de dados foi realizada por meio de banco de dados, onde não foram disponibilizados os dados de identificação dos participantes, conforme pode-se confirmar no documento: https://sei.prf.gov.br/sei/controlador_externo.php?acao=documento_conferir\&id_orgao_acesso_externo=0, código verificador 15157146, CRC 805CCB39, esta pesquisa não submetida a apreciação de Comitê de Ética em Pesquisa, em acordo com a Resolução n. 510 de 07 de Abril de 2016 do Conselho Nacional de Saúde (CNS) no seu Artigo 1 Inciso V.

A medida da acuidade visual, as medidas de visão cromática, reação ao ofuscamento e estereopsia se deram através do aparelho RZ2000 Plus, certificado pela ANVISA.

A análise estatística de dados foi realizada através do software Microsoft Excel e BioEstat 5.0, onde foram feitas medidas de tendência central e dispersão para os dados quantitativos, e frequência relativa e absoluta para todos os dados. Não foram realizados quaisquer cálculos de validação amostral por ser estudo com a totalidade de prontuários disponibilizados.

As principais limitações da presente pesquisa encontram-se nos fatos de ser uma população selecionada num horário específico e dia pontual de tráfego nas BR-316 e BR-163 em proximidade com o município de Benevides-PA e Santarém-PA, respectivamente, nas datas de Maio e Outubro de 2018. No entanto, por ser população que se caracteriza por grande mobilidade a dos caminhoneiros, este constitui um dos métodos mais factíveis de se discriminar de forma epidemiológica este seguimento profissional.

\section{RESULTADOS}

Neste estudo, 185 prontuários foram avaliados, 99,5\% eram do sexo masculino. Destes, $22,2 \%$ tem idade superior a 50 anos, 28,1\% tem idade entre 40 e 50 anos, $35,1 \%$ estão entre 30 e 40 anos e $11,9 \%$ são menores de 30 anos.

Quanto à escolaridade, $23(12,4 \%)$ apresentam ensino fundamental incompleto, $46(24,9 \%)$ ensino fundamental completo, $6(3,2 \%)$ ensino médio incompleto, $96(51,9 \%)$ ensino médio completo, $7(3,8 \%)$ ensino superior incompleto, $5(2,7 \%)$ ensino superior completo e $1(0,5 \%)$ analfabeto. Um prontuário não foi informado quanto à esta categoria.

Com relação ao tempo de habilitação, a média dos motoristas é de 19,3 anos (desvio-padrão 11,1 anos), com amplitude de 3 a 55 anos, porém, a maior predominância é de $40 \%$ em indivíduos com mais de 20 anos (Tabela 1). 
De acordo com o artigo 147 do Código de Trânsito Brasileiro, encaixa-se na categoria A os condutores de veículos motorizados de duas ou três rodas, com ou sem carro lateral. Já na categoria $D$, são veículos usados no transporte de passageiros cuja lotação exceda oito lugares, excluído o do motorista, e na categoria $E$, os condutores de combinação de veículos em que a unidade tratora se enquadre nas categorias $B, C$ ou $D$ e cuja unidade acoplada, reboque, semirreboque, trailer ou articulada tenha $6.000 \mathrm{Kg}$ ou mais de peso bruto total, ou cuja lotação exceda 8 lugares. Diante disso, 42,7\% dos motoristas dispõem de $\mathrm{CNH}$ na categoria "AE" e 32,4\% estão enquadrados na categoria "AD" (Tabela 1).

Tabela 1 - Distribuição dos motoristas de acordo com Tempo de habilitação e Categoria da carteira de habilitação.

\begin{tabular}{clll}
\hline & & N & $\%$ \\
\hline TEMPO DE & & & \\
HABILITAÇÃO & & 33 & $17,8 \%$ \\
& $<10$ & 69 & $37,3 \%$ \\
& $10 \mathrm{~A} 20$ & 74 & $40,0 \%$ \\
& $>20$ & 9 & $4,9 \%$ \\
\hline CATEGORIA DA & NINF & & \\
CARTEIRA & & 3 & $1,6 \%$ \\
& $\mathrm{C}$ & 6 & $3,2 \%$ \\
& $\mathrm{D}$ & 20 & $10,8 \%$ \\
& $\mathrm{E}$ & 9 & $4,9 \%$ \\
& $\mathrm{AC}$ & 60 & $32,4 \%$ \\
& $\mathrm{AD}$ & 79 & $42,7 \%$ \\
& $\mathrm{AE}$ & 8 & $4,3 \%$ \\
\hline & $\mathrm{NI}$ & 185 & $100 \%$ \\
\hline
\end{tabular}

Fonte: Banco de dados Polícia Rodoviária Federal (PRF), 2018.

Neste estudo pelo menos $27,6 \%$ dos motoristas estavam conduzindo veículos de grande porte sem as condições mínimas visuais exigidas pelo Código de Trânsito Brasileiro, que estabelece pelo menos 20/30 de visão em ambos os olhos. Somado a isso, $23,8 \%$ demonstraram alteração no teste para estereopsia e 76,2\% não demonstraram alteração (Tabela 2).

Tabela 2 - Distribuição dos motoristas quanto à acuidade visual e estereopsia.

\begin{tabular}{cccccc}
\hline VARIÁVEL & N & $\%$ & N & $\%$ \\
\hline ACUIDADE VISUAL & & OD & & & OE \\
\hline $20 / 20$ & 34 & $18,4 \%$ & 41 & $22,2 \%$ \\
$20 / 25$ & 83 & $44,9 \%$ & 70 & $37,8 \%$ \\
$20 / 30$ & 20 & $10,8 \%$ & 24 & $13,0 \%$ \\
$20 / 40$ & 9 & $4,9 \%$ & 14 & $7,6 \%$ \\
$20 / 50$ & 9 & $4,9 \%$ & 14 & $7,6 \%$ \\
$20 / 60$ & 3 & $1,6 \%$ & 1 & $0,5 \%$ \\
$20 / 80$ & 5 & $2,7 \%$ & 2 & $1,1 \%$ \\
$20 / 100$ & 18 & $9,7 \%$ & 20 & $10,8 \%$ \\
NINF & 4 & $2,2 \%$ & 4 & $2,2 \%$ \\
\hline & & & & & \\
ESTEREOPSIA & SIM & 44 & $23,8 \%$ & & \\
& NÃO & 141 & $76,2 \%$ & &
\end{tabular}

Fonte: Banco de dados Polícia Rodoviária Federal (PRF), 2018. 
Outro parâmetro utilizado para avaliar a saúde visual dos condutores é a adequada visão cromática destes, mostrada na TABELA 3. Neste estudo, $88,1 \%$ dos motoristas conseguiam distinguir as cores verde, amarelo e vermelho, os demais apresentaram screening positivo para algum tipo de discromatopsia.

Tabela 3 - Distribuição dos motoristas quanto à visão de cores.

\begin{tabular}{cccc}
\hline VISÃO CROMÁTICA & N & $\%$ \\
\hline VERDE & SIM & 162 & $87,6 \%$ \\
AMARELO & NÃO & 23 & $12,4 \%$ \\
& SIM & 164 & $88,6 \%$ \\
VERMELHO & NÃO & 21 & $11,4 \%$ \\
& SIM & 163 & $88,1 \%$ \\
& NÃO & 22 & $11,9 \%$ \\
\hline
\end{tabular}

Fonte: Banco de dados Polícia Rodoviária Federal (PRF), 2018.

\section{DISCUSSÃO}

Os resultados apontam o predomínio dos caminhoneiros do sexo masculino, com idade média de 41,1 anos e com tempo médio de habilitação maior de 20 anos. De acordo com dados do Departamento Nacional de Trânsito (DENATRAN), dos 45 milhões de motoristas no Brasil, quase 30 milhões são do sexo masculino. Pesquisas indicam que $71 \%$ dos acidentes provocados no país são por homens. Essa relação pode estar associada ao tempo de sono, ao cansaço, às longas jornadas de trabalho, o que pode afetar a saúde física e mental desses profissionais. (RIBEIRO CLS, 2017)

Hoje, segundo dados publicados pelo Instituto Brasileiro de Planejamento e Tributação (IBPT), aproximadamente 41,2 milhões de veículos fazem parte da frota brasileira em circulação, sendo 2 milhões classificados como veículos pesados. O Código Nacional de trânsito já passou por diversas modificações e atualmente estabelece que esses condutores devem passar por exames médicos, incluindo os oftalmológicos, a cada 5 anos para renovação da CNH. (DUARTE WR et al, 2002) Entretanto, de acordo com a nosso estudo, $27,6 \%$ dos condutores avaliados estão dirigindo nas estradas sem as condições necessárias, o que requer uma atenção maior a este tema.

De acordo com SEDANO GS, et al (2010), após analisar um estudo, notou-se que $38,2 \%$ dos caminhoneiros entrevistados nunca haviam realizado um check-up médico, sendo que, 19,6\% nunca foram ao oftalmologista para uma consulta e $9,8 \%$ não procuram este profissional há mais de cinco anos. O principal motivo alegado é a mais absoluta falta de tempo.

A ausência do homem nos serviços de atenção básica, o medo de perder seu trabalho e o déficit de comportamentos preventivos de autocuidado, são questões que contribuem para os preocupantes dados em relação à saúde dos caminhoneiros (HINO P et al 2017).

Diante do exposto, destaca-se a importância da intensificação de ações preventivas com enfoque à saúde e, principalmente, a saúde visual. Além de priorizar a constante qualificação dos profissionais responsáveis pelas triagens de cunho médico no exame de renovação da CNH (DUARTE WR et al, 2002).

\section{CONCLUSÃO}

Considera-se que este estudo avança ao quantificar a acuidade visual dos condutores de trânsito de veículos pesados nas condições em que se encontram no momento da direção. É de extrema importância a elaboração de novas pesquisas no intuito de caracterizar melhor os fatores e hábitos de vida dos motoristas que influenciam sua saúde visual. Por fim, baixa acuidade visual ainda é bastante prevalente entre motoristas profissionais, podendo estar ligada aos acidentes no trânsito nas rodovias do Estado do Pará. Elaborar programas de saúde pública que atinjam esse público-alvo, que é, por natureza, itinerante, constitui grande desafio ao Sistema Único de Saúde que hoje é totalmente estruturado para atingir a população de uma forma regionalizada, ou seja, funcional para aqueles que tem residência fixa somente. 


\section{REFERÊNCIAS}

1. BRANDÃO EO, OLIVEIRA NM, PEREIRA MVC et al. Acuidade visual de motoristas de veículos pesados numa rodovia de grande circulação - Problemática e sugestões. Arq Bras Oftal 58(2), ABRIL/1995.

2. DENATRAN. Resolução № 425 CONTRAN. (2012). Acesso em 17 de Janeiro de 2019, disponível em https://www.denatran.gov.br/download/Resolucoes/(Resolu\%C3\%A7\%C3\%A30\%20425.-1).pdf

3. DUARTE WR, DUARTE MCR, PARANHOS C, et al. O perfil oftalmológico dos candidatos à carteira de motorista na cidade de Pelotas - RS. Arq Bras de Oftalmol 2002;65:651-4.

4. HINO P, FRANCISCO TR, ONOFRE PSC et al. Análise à saúde de caminhoneiros. Ver enferm UFPE online, Recife, 11(Supl. 11):4741-8, nov., 2017.

5. QUAGLIATO LB, SOARES CBCC, SOARES MVCC et al. Avaliação oftalmológica de um grupo de motoristas profissionais de Campinas, São Paulo. Rev Med (São Paulo). 2012 out-dez.;91(4):261-6.

6. RIBEIRO CLS, NUNES JD, OLIVEIRA DML. Caracterização da saúde dos condutores de veículos pesados e perigosos que trafegam em rodovia federal no Estado de Sergipe. Ciências biológicas e de saúde unit. V.4. N.2. p. 35-52. Out. 2017.

7. SEDANO GS, FERREIRA SCM, VALENTE GSCV et al. Educação em saúde: Um desafio do enfermeiro do trabalho na atenção à saúde dos caminhoneiros. Rev. Pesqui. Cuid. Fundam. (Online);2(2):760-769, abr-jun. 2010. 